\title{
Reproductive and productive recall of set inclusion information
}

\author{
RICHARD A. GRIGGS \\ University of Florida, Gainesville, Florida $\$ 2611$
}

\begin{abstract}
Subjects studied four different paragraphs, each describing a set inclusion structure, and then were tested for their memory of the information in the paragraphs (reproductive recall) and information deducible from the paragraphs (productive recall). The results showed reproductive recall to be superior to productive recall, but even reproductive recall was poor. Furthermore, subjects often failed to generate deductions in recall even though the information necessary to permit such deductions had been recalled. The results are in general agreement with previous findings on the processing of set inclusion information given in text but conflict with those of recent studies on processing other types of meaningful prose. The set inclusion relation appears to present special difficulties for the integration of related information in prose.
\end{abstract}

Frase $(1969,1970)$ investigated the problem of learning from text material and going beyond the information given in the text. Assuming that learning from text is a consequence of establishing relations among pairs of items, Frase developed an experimental method for studying the processing of text. Using sets as the units of text analysis, Frase employed paragraphs that contained sentences which asserted relations between five sets. If $A, B, C, D$, and $E$ represent the sets, then the four primary sentences of the paragraph symbolically were All As are Bs, All Bs are Cs, All Cs are Ds, and All Ds are Es. Thus, the basic text structure of a paragraph was a series of subset relations, a set inclusion relationship. Such stimulus material permits deducible information (going beyond the text-All As are Cs, All Bs are Ds, and so on). Frase refers to memory for presented information as reproductive memory and to memory for deducible information as productive memory.

Subjects in Frase's $(1969,1970)$ experiments were asked to read paragraphs in order to decide if the statement typed at the top of each paragraph was a valid conclusion from the text or, in some experiments, in order to underline the information in the paragraph that was needed to verify the conclusion. This task was followed by a free recall test in which subjects were asked to write down all the assertions in the paragraph they could recall and all inferences they could make from the information in the paragraph.

In Frase (1969), reproductive recall was substantially higher than productive recall, but even reproductive recall was poor. In Study 3, for example, reproductive

I am deeply indebted to Frank Restle for his continued support and assistance. Requests for reprints should be sent to Richard A. Griggs, Department of Psychology, University of Florida, Gainesville, Florida 32611. Frank Restle sponsors this paper and takes full editorial responsibility for it content. recall was $15 \%$ of the possible recall, while productive recall was only $4 \%$. The number of assertions that were recalled was a decreasing function of the number of presented sentences that would have to be related to each other to produce an assertion between terms. That is, an assertion requiring four sentences (e.g., All As are Es) was less likely to be recalled than one requiring three sentences (e.g., All As are Ds), and so on.

Frase (1970) increased the amount of processing of text content by varying the number of conclusions that had to be verified per paragraph. Subjects had to verify two, four, or six conclusions per paragraph. He found that both reproductive and productive recall increased as the amount of processing increased, but that reproductive recall, though not very high, was still much better than productive recall. At the highest level of processing (verifying six conclusions), reproductive recall was only $35 \%$ and productive recall $15 \%$. Productive recall was especially low since the six conclusions that were verified were related to all possible inferences from the text.

Frase's results are inconsistent with those of Potts (1972, 1974, 1975; Scholz \& Potts, 1974), who examined a similar set-theoretic relation. Potts investigated the processing of paragraphs describing linear ordering relations such as $A>B>C>D$, where $A$, $B, C$, and D symbolically represent the terms of the ordering and " $>$ " some comparative adjective describing the dimension on which the terms are ordered. As in Frase's work, only the adjacent pairs in the relation are presented in a paragraph, that is, $\mathrm{A}>\mathrm{B}, \mathrm{B}>\mathrm{C}$, $C>D$. Using a study/test procedure with a true-false test, Potts found that performance (proportion correct or response latency) on productive information was superior to performance on reproductive information. Potts' explanation of these results is that subjects make the permissible inferences (e.g., A $>$ C) during study, integrate these with the presented order information 
to construct a more general semantic representation of the ordering, and store this representation, not a set of the order relations. This agrees with recent findings in sentence memory experiments (e.g., Bransford, Barclay, \& Franks, 1972; Bransford \& Franks, 1971; Cofer, 1973) which support the constructivist theory of language comprehension. Constructivist theory argues that humans do not store meaningful verbal material verbatim or merely deep structure representations of the material but rather actively transform the linguistic input into a cognitive structure which is a joint function of the input information and prior knowledge. Frase's results do not indicate such a constructive process.

Griggs (1976) investigated both set-theoretic relations in the same experimental conditions and found definite processing differences between the two relations, substantiating the earlier observed differences. Using a study/true-false test paradigm, Griggs found that when processing set inclusion information in text, subjects fail to make many valid transitive deductions, and this failure is an increasing function of the distance separating the sets in the inclusion. Subjects do not appear to integrate set inclusion information into a more general semantic structure and store this structure in memory as they do linear ordering information.

The present study investigated set inclusion performance using a self-paced study/free recall test paradigm. No data are available on recall of set inclusion information in a study/test paradigm. Frase $(1969,1970) \mathrm{em}-$ ployed an incidental learning paradigm and Griggs (1976) used a true-false test. Having subjects study the set inclusion paragraphs for as long as they wish and informing them that they are going to be tested on the information contained in the paragraphs seems to be a more natural way of studying text processing.

\section{METHOD}

\section{Subjects}

Subjects were 16 undergraduate students at Indiana University who were fulfilling course requirements for experimental participation.

\section{Materials}

The stimuli consisted of four paragraphs, each of which described a five-term set inclusion. The paragraphs were taken from Griggs (1976) and are similar to those used by Frase $(1969,1970)$.

Each paragraph presented the intended set inclusion as four adjacent relations in a chained order (i.e., AB, BC, CE, DE). Extraneous material was inserted between adjacent pairs to make the paragraph seem more realistic. The content of each paragraph was fictional so that any possible confounding with background knowledge was minimized. The four paragraphs described the natives of a foreign country, space knowledge, the birds of a foreign country, and the production of foreign cars. The following is a sample paragraph:

All the Fundalas are outcasts from other tribes in Central Ugala. These people are isolated from the other tribes because it is the custom in this country to get rid of certain types of people. All the outcasts of Central Ugala are hill people. The hills provide a most accommodating place to live. All the hill people of Central Ugala are farmers. The upper highlands provide excellent soil for cultivation. All the farmers of this country are peace-loving people, which is reflected in their artwork. Altogether, there are about 15 different tribes in this area.

\section{Procedure}

All subjects were run at one time in a large classroom, and no apparatus was necessary. The study and test materials and instructions were printed on separate sheets of paper which were stapled together to form a booklet. Each subject was given one of the booklets and told not to turn any pages in the booklet unless instructed to do so.

Subjects were told that they would read and study four paragraphs for as long as they wanted and then be tested on the information in the paragraphs. No type-of-test information was provided. They were also instructed that each paragraph was on a separate page and that, once they turned a page, they were not to go back. All subjects studied the paragraphs in the same order. A detailed set of instructions about the recall task followed the four paragraphs in the booklet. Subjects were told to recall, in any order, as much information about a particular paragraph as possible. They were told to recall not only pairwise relations that had been presented in the paragraph but also any relations that could be deduced from the information in the paragraph. An example of a deducible relation using the universal quantifier "All" was provided, as well as instructions about the way their recall should be structured-simple declarative sentences, each relating two sets. The recall tests were encountered in the same order as presentation. The title of each paragraph was provided on the recall sheet for that paragraph. Subjects were allowed as much time as they wanted for recall.

\section{RESULTS}

Recall protocols were scored for the presented assertions and all possible deductions. Three judges scored the protocols and agreement of at least two judges was obtained on all recall responses. As in Frase $(1969,1970)$, recall measures were adjusted for subjects' tendency to guess at relations. Incorrect recall was subtracted from correct recall. For example, if a subject said that All As are Bs and that All Bs are As, he would not get credit for All As are Bs. There was not a great deal of difference between corrected and uncorrected scores.

Recall protocols were scored by two methods. Using Method 1, only recall having a correct relation and using the universal quantifier was scored as correct. For Method 2, recall having a correct relation, whether or not the universal quantifier was used, was scored as correct (e.g., As are Bs or All As are Bs was accepted as correct).

Both level of recall and the difference between reproductive and productive recall were the same for all four paragraphs, so the following results are for the four paragraphs combined. As in Frase (1969), recall was a decreasing function of the number of assertions that had to be related to each other to produce a conclusion. The percentage of possible correct responses for reproductive and productive recall was calculated by both scoring methods. The results are given in Table 1 .

Reproductive recall was better than productive recall by both scoring methods. To test the generalizability of 
Table 1

Percentage of Possible Correct Responses for Reproductive and Productive Recall Using Two Scoring Methods

\begin{tabular}{ccc}
\hline $\begin{array}{c}\text { Scoring } \\
\text { Method }\end{array}$ & $\begin{array}{c}\text { Reproductive } \\
\text { Recall }\end{array}$ & $\begin{array}{c}\text { Productive } \\
\text { Recall }\end{array}$ \\
\hline 1 & $30 \%$ & $15 \%$ \\
2 & $50 \%$ & $30 \%$ \\
\hline
\end{tabular}

this superiority, two-tailed sign tests were performed using the reproductive and productive results on each paragraph for each subject as a matched pair. For both scoring methods, reproductive recall was significantly greater than productive recall $(\mathrm{z}=3.8, \mathrm{p}<.001$ and $\mathrm{z}=3.3, \mathrm{p}<.01$, respectively).

Using Method 2, the percentage of correct recall that contained the universal quantifier was calculated. Sixty percent of the reproductive recall contained "All," whereas $51 \%$ of the productive recall did.

The probability of the correct recall of a deduction, given that the assertions necessary to make the deduction were recalled, was also calculated. Using the recall results of Method 2, this probability was found to be .56. Thus, it appears that, even though the information necessary to make deductions was available, subjects to a large extent did not utilize this information in their recall.

\section{DISCUSSION}

As expected, recall was greater than that observed by Frase $(1969,1970)$, but it was still not very high, even though subjects knew they were going to be tested and self-paced study and recall periods were employed. Frase's finding that reproductive recall was better than productive recall was replicated. Using the more lenient Method 2, reproductive recall was $50 \%$ of the possible recall and productive recall was only $30 \%$. Brockway, Chmielewski, and Cofer (1974) have also found evidence for poor reproductive recall of set inclusion information. Using three different performance tasks but employing similar set inclusion paragraphs, Brockway et al. found that subjects with instructions to recall only the presented material could recall only 1.13 and 1.63 sentences (both out of a possible 4.0) for the two paragraphs studied. This small amount of recall agrees with both the Frase findings and present results.

The present results are also in agreement with Griggs (1976) in that subjects did not appear to be making the permissible deductions during study and constructing and storing a more general semantic representation of the set inclusion information. Subjects seem to have stored only the material which was actually presented and to have made few deductions at time of test. This is indicated by the small amount of productive recall and the fact that many deductions were not generated in recall, even with instructions to do so and when the necessary information to permit them was recalled. The probability of such recall given the recall of the necessary assertions was only .56. Subjects appeared hesitant to generate deductions. This does not agree with the sentence memory studies or linear ordering research discussed earlier. For linear orderings, performance is better on deductions than on assertions presented in the text. As pointed out by Frase (1975), the set inclusion relation appears to create special difficulties for the integration of the presented and deducible information into a more general semantic structure. The next step is to identify the reasons for this.

\section{REFERENCES}

Bransford, J. D., Barclay, J. R., \& Franks, J. J. Sentence memory: A constructive versus interpretive approach. Cognitive Psychology, 1972, 3, 193-209.

Bransford, J. D., \& Franks, J. J. The abstraction of linguistic ideas. Cognitive Psychology, 1971, 2, 331-350.

Brockway, J., Chmielewski, D., \& Cofer, C. N. Remembering prose: Productivity and accuracy constraints in recognition memory. Journal of Verbal Learning and Verbal Behavior, 1974, 13, 194-208.

Cofer, C. N. Constructive processes in memory. American Scientist, 1973, 61, 537-543.

Frase, L. T. Structural analysis of the knowledge that results from thinking about text. Journal of Educational Psychology Monograph Supplement, 1969, 60(6, Part 2).

FrASE, L. T. Influence of sentence order and amount of higher level text processing upon reproductive and productive memory. American Education Research Journal, 1970, 7, 307-319.

Frase, L. T. Prose processing. In G. Bower (Ed.), The psychology of learning and motivation (Vol. 9). New York: Academic Press, 1975.

GRIGGs, R. A. Logical processing of set inclusion relations in meaningful text. Memory \& Cognition, 1976, 4, 730-740.

Ports, G. R. Information processing strategies used in the encoding of linear orderings. Journal of Verbal Learning and Verbal Behavior, 1972, 11, 727-740.

Potrs, G. R. Storing and retrieving information about ordered relationships. Journal of Experimental Psychology, 1974, 103, 431-439.

Potrs, G. R. Bringing order to cognitive structures. In F. Restle, R. Shiffrin, J. Castellan, H. Lindman, \& D. Pisoni (Eds.), Cognitive Theory (Vol. 1). Hillsdale, N.J: Lawrence Erlbaum, 1975.

Scholz, K. W., \& Potts, G. R. Cognitive processing of linear orderings. Journal of Experimental Psychology, 1974, 102, 323-326.

(Received for publication September 24, 1976.) 